This is a postprint version of the following published document:

González, M., Martín-Fabiani, I., Baselga, J. y Pozuelo, J. (2011): Magnetic nanocomposites based on hydrogenated epoxy resin. Materials Chemistry and Physics, 132 (2-3), pp.: 618-624.

DOI: 10.1016/j.matchemphys.2011.11.077

(c) Elsevier, 2011

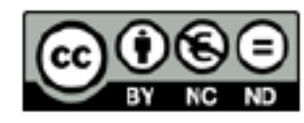

This work is licensed under a Creative Commons Attribution-NonCommercialNoDerivatives 4.0 International License. 


\title{
Magnetic nanocomposites based on hydrogenated epoxy resin
}

\author{
María González, Ignacio Martín-Fabiani, Juan Baselga, Javier Pozuelo * \\ Departamento de Ciencia e Ingeniería de Materiales e Ingeniería Química (IAAB), Universidad Carlos III de Madrid, 28911 Leganés, Madrid, Spain
}

\begin{abstract}
A hydrogenated derivative of diglycidylether of bisphenol A (HDGEBA) has been successfully employed to prepare magnetic thermosetting nanocomposites. Magnetite nanoparticles of $\approx 10 \mathrm{~nm}$ modified with oleic acid were synthesized by co-precipitation of ferric and ferrous salts in ammonium solutions. Nanocomposites with amounts up to $5 \% w^{-1}$ of nanoparticles were dispersed in HDGEBA by co-solvent method and cured with m-xylilendiamine. Both the particles and nanocomposites were characterized by FTIR, DSC, TGA, XRD, TEM, DMTA and VSM. Due to the lower polarity of the hydrogenated resin, the enhanced compatibility with magnetite oleic coverage allowed obtaining good dispersions of the nanoparticles for compositions up $1 \% \mathrm{ww}^{-1}$, while at higher concentration agglomerates were observed. Two behaviors in the thermal properties were obtained: for compositions below $1 \%$ $\mathrm{ww}^{-1}$ the glass tran-sition temperature increased with the amount of nanoparticles, whereas for higher amounts the glass transition temperature decreased. Superparamagnetic behavior was observed in both the particles and the nanocomposites; the blockage temperature gradually increases with loading but it is lower than the observed for bulk magnetite nanoparticles. This fact reflects that nanoparticle-nanoparticle distances in the nanocomposites gradually decrease with loading which is in accordance with a good dispersion state for low loadings and the presence of aggregates at high loadings.
\end{abstract}

Keywords: Nanocomposite Magnetite Epoxy

\section{Introduction}

Design, development and manufacture of nanostructured materials with specific functional properties is an active research field with growing interest in recent years. Incorporation of nanoparticles into polymer matrices is a good strategy to transfer functional properties of particles to polymers. Nanocomposites prepared in this way are an important class of materials which combines both the functional properties of the nanoparticles and the advantages of polymer matrices. There are excellent reviews [1-3] that show significant improvements in mechanical, electrical and optical properties of materials when relatively small amounts of nanoparticles are incorporated in polymers [4-7]. Since the type, size and geometry of nanoparticles (spheres, sheets, tubes, bars, etc.) plays an important role on the properties of the final material, one of the most important challenges is to control these properties during the nanoparticles preparation. Nevertheless the key factor for the material performance is to obtain a homogeneous distribution of nanoparticles within polymer matrices, avoiding the formation of agglomerates, which is a general problem on the preparation of nanocomposites.

\footnotetext{
* Corresponding author. Tel.: +3491 6249940; fax: +34 916249430. E-mail address: jpozue@ing.uc3m.es (J. Pozuelo).
}

To control the size of nanoparticles, both the thermodynamics of nuclei formation and diffusional processes during the growth must be controlled. Conditions must be optimized to: (i) ensure the formation of a large number of nuclei and (ii) hinder diffusion to inhibit crystal growth. For the first, high concentrations of precursors are used, while diffusion can be controlled carrying out the synthesis in confined environments such as gels, solids or micellar systems [8]. The synthesis of ferrite nanoparticles has been intensively investigated in recent years because of their electrical and magnetic properties. Many procedures have been explored: chemical coprecipitation [9-11], co-precipitation in reverse microemulsions [12,13], hydrothermal synthesis, sol-gel techniques [14], use of citrate precursors [15], mechanical mixing [16] and thermal decomposition in solid matrices [17] are some examples. Nevertheless, the simplest and cheapest ways to obtain nano-sized ferrites are chemical co-precipitation and the use of inverse microemulsions.

The study of magnetic nanoparticles has a strong interest because of their huge potential applications, among which medical applications like hyperthermal treatments for cancer control [18-23] and technological applications, such as the development of effective shielding materials for high frequency electromagnetic radiation [24] can be highlighted.

The effectiveness of magnetic nanoparticles in shielding applications is related with dissipation of electromagnetic radiation. Chung [25] defines three mechanisms for electromagnetic 
interference processes: reflection, absorption and multiple reflections. The first one is important in metallic materials, while multiple reflections are important in polymeric foams or porous materials. Absorption of electromagnetic radiation by magnetic particles induces magnetic spin rotation and the magnetic energy can be dissipated by heat transfer to the matrix. This is the most appropriate mechanism for protecting the internal elements of an electronic device. In general, absorption losses are proportional to the product $\sigma_{\mathrm{r}} \mu_{\mathrm{r}}$ (where $\sigma_{\mathrm{r}}$ and $\mu_{\mathrm{r}}$ are the relative electrical conductivity and relative permeability respectively), while reflection losses are proportional to the ratio $\sigma_{\mathrm{r}} / \mu_{\mathrm{r}}$. Therefore, particles with high magnetic permeability as magnetic ferrites are suitable for these processes.

Current research focuses on the use of nano-sized particles because of the following reasons: (i) To increase the effectiveness of electromagnetic absorption. It is known that electrical conductivity occurs on the surface of the particles (skin effect). Since the skin thickness is inversely proportional to the radiation frequency, the skin thickness for absorbing radiation in the range of gigahertz is a few nanometers. Therefore the specific surface fraction (and so absorption effectiveness) can be increased reducing particle size; (ii) the superparamagnetism phenomenon. Superparamagnetic nanocomposites are materials in which monodomain ferromagnetic nanoparticles with sizes under $50 \mathrm{~nm}$, are well dispersed in a solid matrix. At room temperature, thermal energy is enough to maintain a random distribution of the magnetic moments of the nanoparticles [26]. But these materials show a blocking temperature below which thermal energy is not able to orientate randomly the magnetic moments and polarization appears in the presence of low magnetic fields [27]. In addition, as Kolev et al. showed, the absorption frequency increases as the nanoparticle size is decreased [28]. The most commonly used magnetic nanoparticles are ferrites. However, their main drawback is that they tend to form agglomerates preventing homogeneous distribution of particles within the polymers and, as a consequence, loosing their superparamagnetic behavior and affecting the nanocomposites mechanical properties. One possible solution is the use of surfactants which can act as compatibilizers between the particles and the matrix allowing also to control the particle size during the synthesis.

Polymers are very attractive matrices for embedding magnetic particles due to their thermal and electrical properties, density, processability and price. Gyergyek et al. [29] classified the methods for preparing magnetic polymer matrix nanocomposites in three groups: (i) synthesis of magnetic nanoparticles in a polymer matrix or on its surface [30,31]; (ii) polymerization reaction in the presence of nanoparticles [32]; (iii) mixing polymers and nanoparticles [33,34]. Methods (i) and (ii) do not allow an adequate control of particle size and the variety of nanocomposites that can be prepared is considerably limited; using method (iii), which is the most versatile, particles can agglomerate easily when mixing with the matrix unless their surface is modified. Nanocomposites of magnetite or maghemite with a wide range of different thermoplastic polymers, from conventional polymers such as polypropylene [35], polystyrene [36-38], or poly(methyl methacrylate) [39], to biodegradable polymers $[40,41]$ or even conducting polymers [42] have been prepared this way.

Preparation and characterization of magnetic nanocomposites with thermosetting polymers is scarcely reported in the literature. Uncoated $\mathrm{Fe}_{3} \mathrm{O}_{4}$ nanoparticles have been used in epoxy matrices based on diglycidylether of bisphenol A (DGEBA) [43-45], but the cured materials showed poor dispersion of the particles and big aggregates were observed even at low particle loading. The use of silanized $\mathrm{Fe}_{3} \mathrm{O}_{4}$ particles seems to improve dispersion, at least in photocurable epoxy formulations [46]. In this work a thermosetting polymer based on epoxy resin has been selected as nanocomposite matrix. Epoxy resins are widely used as adhesives, coatings and matrix in polymer composites due to its low viscosity, good insulating properties even at high temperatures and good chemical and thermal resistance [47]. They are also used as encapsulants for electronic devices; incorporation of magnetite particles may lead to the development of encapsulant formulations with electromagnetic shielding properties.

We have selected a hydrogenated derivative of diglycidylether of bisphenol A (HDGEBA) epoxy resin, whose main feature is the absence of phenyl groups in its structure while keeping good reactivity towards common amine based curing agents. Its low polarity and the presence of flexible cyclohexyl groups instead of rigid aromatic rings makes the viscosity of HDGEBA appreciably lower than DGEBA based resins, making it especially interesting for coatings, shaping complex components and encapsulation. Additionally, it is expected to be more compatible with aliphatic organic structures like the hydrophobic chains of surfactants. Up to the authors' knowledge, the use of this resin has only been reported in a few patents [48-51].

In the present work magnetite nanoparticles of about $10 \mathrm{~nm}$ in diameter modified with oleic acid have been synthesized by co-precipitation of ferric and ferrous chlorides in ammonium solution. Thermosetting nanocomposites based on HDGEBA have been prepared at different nanoparticle loading. Both the particles and nanocomposites have been characterized by FTIR, TGA, XRD, TEM, DSC, DMTA and VSM. Results of this work will be used in an on-going work to evaluate the applicability of these materials as electromagnetic protective panels in the range of $\mathrm{GHz}$.

\section{Experimental}

\subsection{Materials}

Ferrous chloride tetrahydrate, ferric chloride hexahydrate, ammonium hydroxide $(28 \% \mathrm{v} / \mathrm{v})$, oleic acid, and tetrahydrofurane were purchased from Sigma-Aldrich and used without further purification. The hydrogenated derivative of diglycidyl ether of bisphenol-A (HDGEBA) was supplied by CVC Specialty Chemicals (USA), with a mass per epoxy groups mol of $210 \mathrm{~g} \mathrm{~mol}^{-1}$, determined by acid titration. m-xylilenediamine (Sigma-Aldrich) was used as curing agent.

\subsection{Synthesis of nanoparticles}

Magnetite nanoparticles were prepared according to a previously reported method [9-11]: $5.80 \mathrm{~g}$ of $\mathrm{FeCl}_{3} \cdot 6 \mathrm{H}_{2} \mathrm{O}$ and $2.15 \mathrm{~g}$ of $\mathrm{FeCl}_{2} \cdot 4 \mathrm{H}_{2} \mathrm{O}$ were dissolved in $200 \mathrm{~mL}$ of deionized water in a round bottom flask placed in an ultrasonic bath with mechanical stirring at $70-80^{\circ} \mathrm{C}$. $10 \mathrm{~mL}$ of $25 \% \mathrm{NH}_{4} \mathrm{OH}$ were quickly added to the solution. After a few seconds the solution turned black (typical magnetite color) and $10 \mathrm{~mL}$ of oleic acid were added to the suspension stirring vigorously for $2 \mathrm{~h}$. The black fine magnetite precipitate was separated from the solution using a magnet and washed several times with hot deionized water and acetone to remove non reacted metallic salts and excess of oleic acid respectively. The clean precipitate was dried in vacuum at $100^{\circ} \mathrm{C}$ during $24 \mathrm{~h}$. This process yields magnetite nanoparticles covered by an almost monolayer of oleic acid.

\subsection{Preparation of nanocomposites}

Epoxy nanocomposites with different amounts of nanoparticles $\left(0.25,0.5,1,2.5\right.$ and $\left.5 \% \mathrm{w} \mathrm{w}^{-1}\right)$ were prepared via co-solvent mixtures. Both the nanoparticles and HDGEBA were previously blended with THF and appropriate amounts of the mixtures were placed in a glass vial, mechanically stirred and sonicated at room 
temperature. It is worthy to note that suspensions of the nanoparticles in THF:HDGEBA were stable. Solvent was removed in vacuum at $80^{\circ} \mathrm{C}$ prior curing and stable dispersions of nanoparticles on HDGEBA were obtained. Dispersions were mixed with stoichiometric amounts of $m$-xylilenediamine (curing agent for HDGEBA) and cured at $90^{\circ} \mathrm{C}$ for $1 \mathrm{~h}$. Samples were postcured $1 \mathrm{~h}$ at $120^{\circ} \mathrm{C}$ and $1 \mathrm{~h}$ at $130^{\circ} \mathrm{C}$ to ensure full conversion.

\subsection{Measurements}

Dynamic Light Scattering (DLS) measurements were carried out in a Malvern Zetasizer Nano-ZS instrument. Fourier Transform Infrared Spectroscopy in the mid range (FT-IR, PerkinElmer GX 2000) was used to characterize the synthesized nanoparticles. Thermo Gravimetric Analysis (TGA, PerkinElmer STA 6000 system) and Wide-Angle X-Ray Diffraction (XRD, Panalytical X'pert Pro X-ray diffractometer) were performed on both the synthesized particles and the nanocomposites. TGA measurements were carried out in nitrogen atmosphere from $50^{\circ} \mathrm{C}$ to $600^{\circ} \mathrm{C}$ with a heating rate of $10^{\circ} \mathrm{C} \mathrm{min}-1$. Magnetic properties of the particles and composites were investigated by Vibrating Sample Magnetometry (VSM, CFMS Cryogenic Ltd) at $2 \mathrm{~K}$ and $300 \mathrm{~K}$ with a $10 \mathrm{~T}$ magnetic field. FC (field cooling) and ZFC (zero field cooling) curves were obtained from $2 \mathrm{~K}$ to $300 \mathrm{~K}$. Morphology of all the prepared nanocomposites was observed by Transmission Electron Microscopy (TEM, $200 \mathrm{kV}$ Philips Tecnai 20). The glass transition temperature of the samples was measured by Differential Scanning Calorimetry (DSC, Mettler Toledo 822 differential scanning calorimeter equipped with a liquid nitrogen reservoir) at a heating rate of $10^{\circ} \mathrm{C} \mathrm{min}^{-1}$. Mechanical characterization was performed with a Dynamic Mechanical Thermal Analyzer (DMTA, TA Instruments Q800) at $1 \mathrm{~Hz}$.

\section{Characterization}

\subsection{Nanoparticles characterization}

Stable dispersions of oleic covered magnetite nanoparticles were obtained when using THF as solvent. Dynamic light scattering (DLS) measurements on these dispersions showed a narrow size distribution of particles centred at $16 \mathrm{~nm}$. No features with size above $50 \mathrm{~nm}$ were observed, so the nanoparticles can be expected to be magnetic monodomains. Particle size as measured by DLS is obtained from the translational diffusion coefficient through the Stokes-Einstein equation for Brownian motion. Therefore, the measured size corresponds to the size of an equivalent hard sphere formed by the magnetite core plus the oleic layer probably swelled with solvent.

The oleic acid amount on the surface of the nanoparticles was determined by thermogravimetric analysis. Three weight losses were observed in the TGA thermogram (Fig. 1). The first weight loss is very small and appears at around $100^{\circ} \mathrm{C}(\approx 2.4 \%)$. It can be attributed to water absorbed on the nanoparticle surface stabilized by the presence of oleate groups and the hydrophilicity of nanoparticles surface. The second loss, at around $250^{\circ} \mathrm{C}$, could be due to physisorbed oleic acid $(\approx 4.5 \%)$ while the loss centred at $370^{\circ} \mathrm{C}$ can be attributed to oleic acid chemisorbed as oleate. The total oleic acid amount covering the nanoparticles determined by TGA is $18.6 \%$ $\left(\mathrm{w} \mathrm{w}^{-1}\right)$. This amount corresponds to a monolayer of oleic acid on the surface of the particles.

Infrared spectrum in the mid range $\left(400-4000 \mathrm{~cm}^{-1}\right)$ of the nanoparticles is shown in Fig. 2 (spectrum of the neat oleic acid is also presented for comparison). Typical carboxylate asymmetric tension bands are observed at 1525 and $1603 \mathrm{~cm}^{-1}$, indicating that oleic acid in carboxylate form is chemisorbed on the surface of oxide particles. The band centred at $1706 \mathrm{~cm}^{-1}$ corresponds to

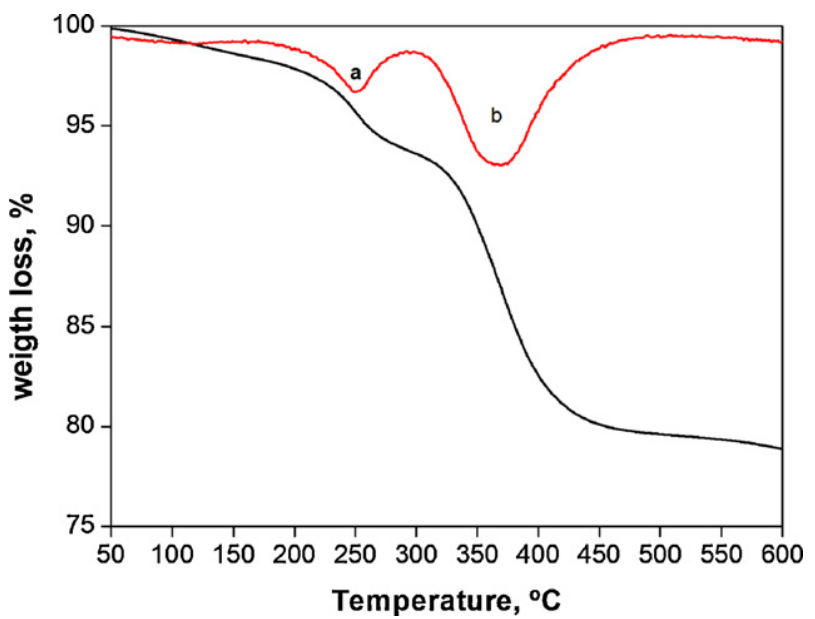

Fig. 1. TGA of magnetite nanoparticles coated with oleic acid.

$\mathrm{C}=\mathrm{O}$ tension of carboxylic acid and the band at $1430 \mathrm{~cm}^{-1}$ can be attributed to in plane deformation of coordinated hydroxyl groups of $\mathrm{COOH}$, i.e. oleic acid physisorbed on the nanoparticles surface. The wide band centred at $3400 \mathrm{~cm}^{-1}$ (which is missing in the pure oleic acid spectrum), can be attributed to the $\mathrm{O}-\mathrm{H}$ vibration of adsorbed water on nanoparticle surface in accordance with the water content found by TGA (2.4\%). It is worthy to note a high intensity band in the nanoparticle spectrum centred at $592 \mathrm{~cm}^{-1}$. This absorption is characteristic of $\mathrm{Fe}-\mathrm{O}$ vibration at tetrahedral and octahedral positions in spinel structure and must be underlined as a magnetite existence proof.

The spinel structure was checked by XRD. X-ray spectrum of the synthesized nanoparticles is shown at Fig. 3, where the diffraction signals $(2 \theta=30.2,35.6,43.5,53.8,57.4$ and 63.0$)$ can be attributed to (220), (311), (400), (422), (511) and (440) crystalline planes of magnetite. These diffraction signals are characteristic of the spinel-like structure of the magnetite and no other phases (such as hematite) are observed. A cell parameter of $0.8632 \mathrm{~nm}$ corresponding to a spinel-type cubic cell was obtained. The crystal size was determined using Scherrer's equation:

$D=\frac{k \lambda}{B \cos \theta}$

where $k=0.9$ was used as Scherrer constant, $\lambda$ is the radiation wavelength and $B$ the width at half height of each diffraction signal in

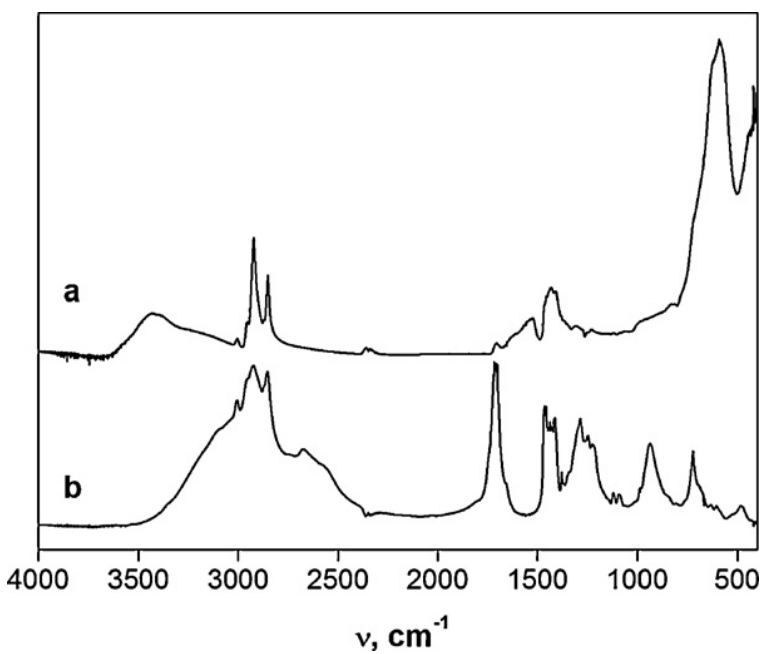

Fig. 2. FTIR spectra of $\mathrm{Fe}_{3} \mathrm{O}_{4}$ nanoparticles coated with oleic acid (a) and pure oleic acid (b). 


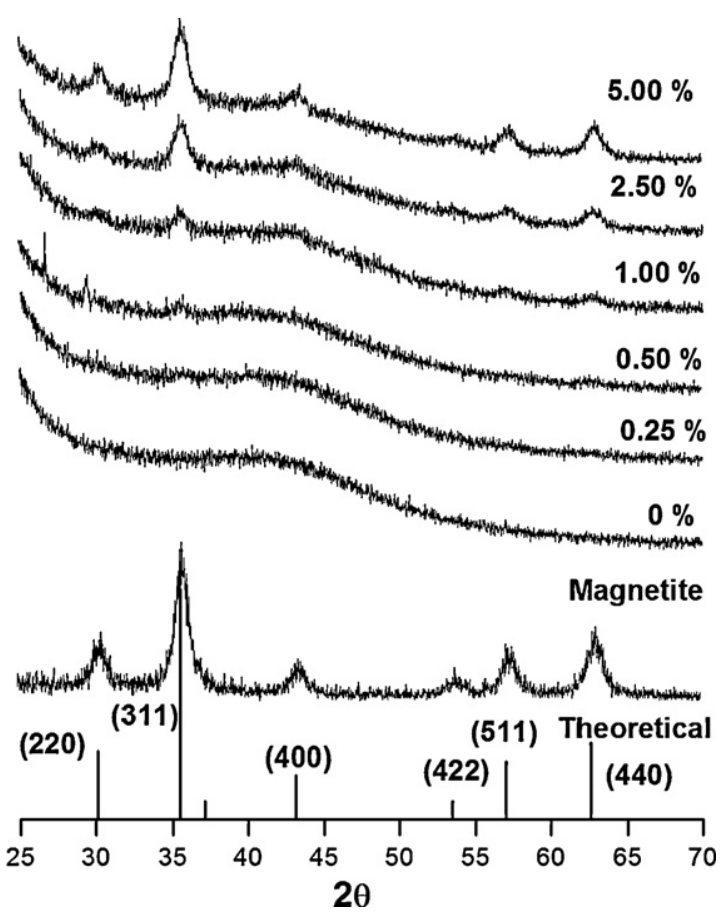

Fig. 3. XRD for all nanocomposites.

radians. According to this equation, and using the diffraction signal corresponding to (311) plane, the crystal size was $9.4 \mathrm{~nm}$.

Nanoparticles size distribution was obtained by TEM. Fig. 4 shows an image of the nanoparticles; the size distribution was obtained from the analysis of several images counting more than 100 nanoparticles. A quite narrow distribution of spherical particles, centred at $9.4 \mathrm{~nm}$, was observed. The size obtained by TEM is identical to the crystal size obtained by XRD $(9.4 \mathrm{~nm})$, indicating that there is no amorphous layer of metal oxides or other metallic impurities surrounding the particle and that the particle is fully crystalline.

The magnetic behavior dependence with temperature was measured under field-cooling $(\mathrm{FC})(\mathrm{H}=100 \mathrm{Oe})$ and zero-fieldcooling (ZFC) conditions (Fig. 5). An irreversible behavior was observed, suggesting a superparamagnetic behavior with a blocking temperature, $T_{\mathrm{B}}$, of $110 \mathrm{~K}$ and a splitting of curves at $150 \mathrm{~K}$. Superparamagnetic behavior was confirmed by hysteresis loops at 2 and $300 \mathrm{~K}$ (Fig. 5). At $300 \mathrm{~K}$, no hysteresis losses were observed

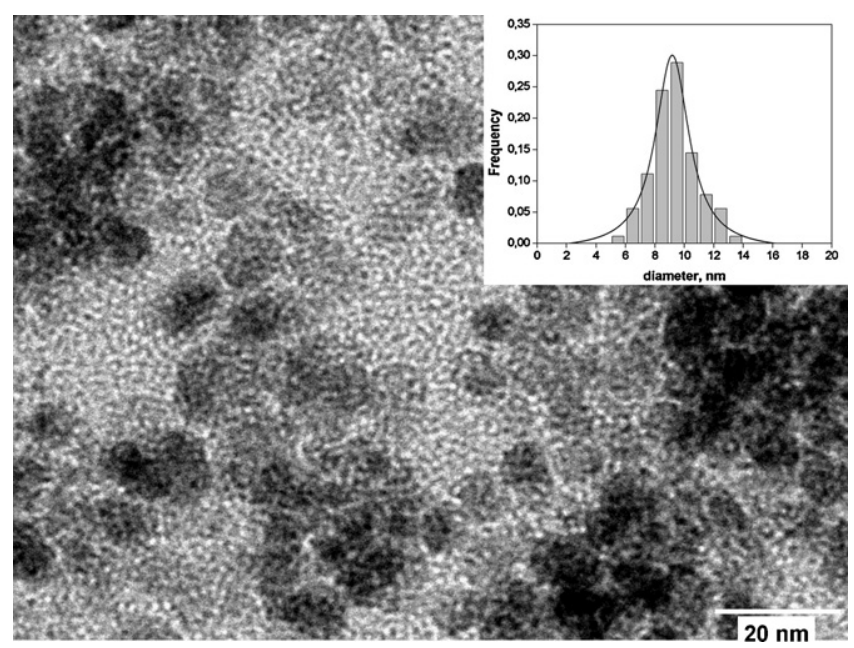

Fig. 4. TEM image of magnetite nanoparticles; insert: particle size distribution.

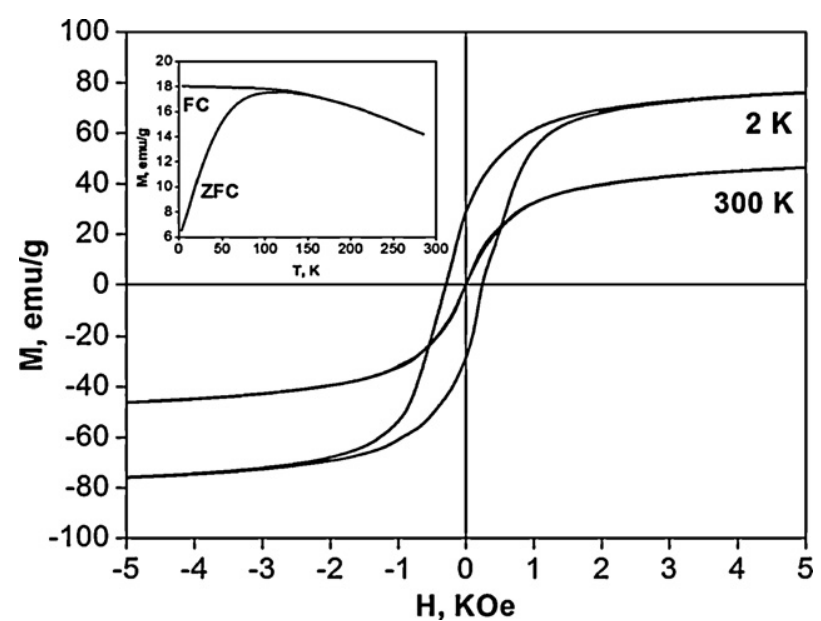

Fig. 5. Hysteresis loops of magnetite nanoparticles at 2 and $300 \mathrm{~K}$. Insert ZFC and FC curves.

with zero remanence and coercitivity. Below $T_{\mathrm{B}}$, hysteresis losses appear in samples. Particles showed a magnetic saturation of 83 and $56 \mathrm{emug}^{-1}$ for temperatures of 2 and $300 \mathrm{~K}$, respectively; a remanence and a coercitive field of $30 \mathrm{emu} \mathrm{g}^{-1}$ and 293 Oe respectively, were observed at $2 \mathrm{~K}$. The difference in magnetic saturation is mainly due to the disorder of nanoparticles spins caused by temperature. From magnetization curves and using Eqs. (2) and (3) proposed by Chantrell et al [52] and Caizer et al. [53], the magnetic diameter and its standard deviation were estimated for a log-normal particle size distribution.

$D=\left[\frac{6 k_{B} T}{\mu_{0} \pi M_{S} H_{0}} \sqrt{\frac{M_{0}}{3 \chi_{i} H_{0}}}\right]^{1 / 3}$

$\sigma=\frac{1}{3}\left[\operatorname{Ln}\left(\frac{3 \chi_{i} H_{0}}{M_{0}}\right)\right]^{1 / 2}$

where $\chi_{i}$ is the initial susceptibility results from the slope in the origin of the magnetization curve, $M_{0}$ and $H_{0}$ result from the extrapolation of the linear portion of the curve $M$ versus $1 / H$ in the saturation domain. Using these expressions, magnetic size and standard deviation were $9.3 \mathrm{~nm}$ and $1.4 \mathrm{~nm}$ respectively, very similar to TEM and XRD results.

\subsection{Nanocomposites characterization}

The X-ray spectra of the nanocomposites (Fig. 3) show the characteristic signals of magnetite nanoparticles and the amorphous halo characteristic of HDGEBA:m-xylilenediamine epoxy system. The diffraction signals of spinel-like structure of magnetite nanoparticles can be clearly observed in nanocomposites containing $1 \%$ magnetite or higher amounts. No displacement of diffraction signals in nanocomposites with respect to pure nanoparticles.

Thermal degradation behavior of the nanocomposites with magnetite was similar to the observed for the neat epoxy matrix (not shown). Thermogravimetric analysis of all the samples showed a degradation temperature around $310^{\circ} \mathrm{C}$, which corresponds to the neat epoxy polymer, although the incorporation of magnetite nanoparticles seems to shift the onset of degradation towards slightly lower temperatures. The degradation of oleic acid is not observed in the thermogravimetric traces, probably due to the low amount of oleic acid in the composites.

The dispersion degree of magnetic nanoparticles was observed by TEM (Fig. 6). Nanocomposites loaded with $0.25 \%$ to $1 \%$ of magnetite showed a good dispersion and very low proportion of agglomerates, although agglomerates were observed in 


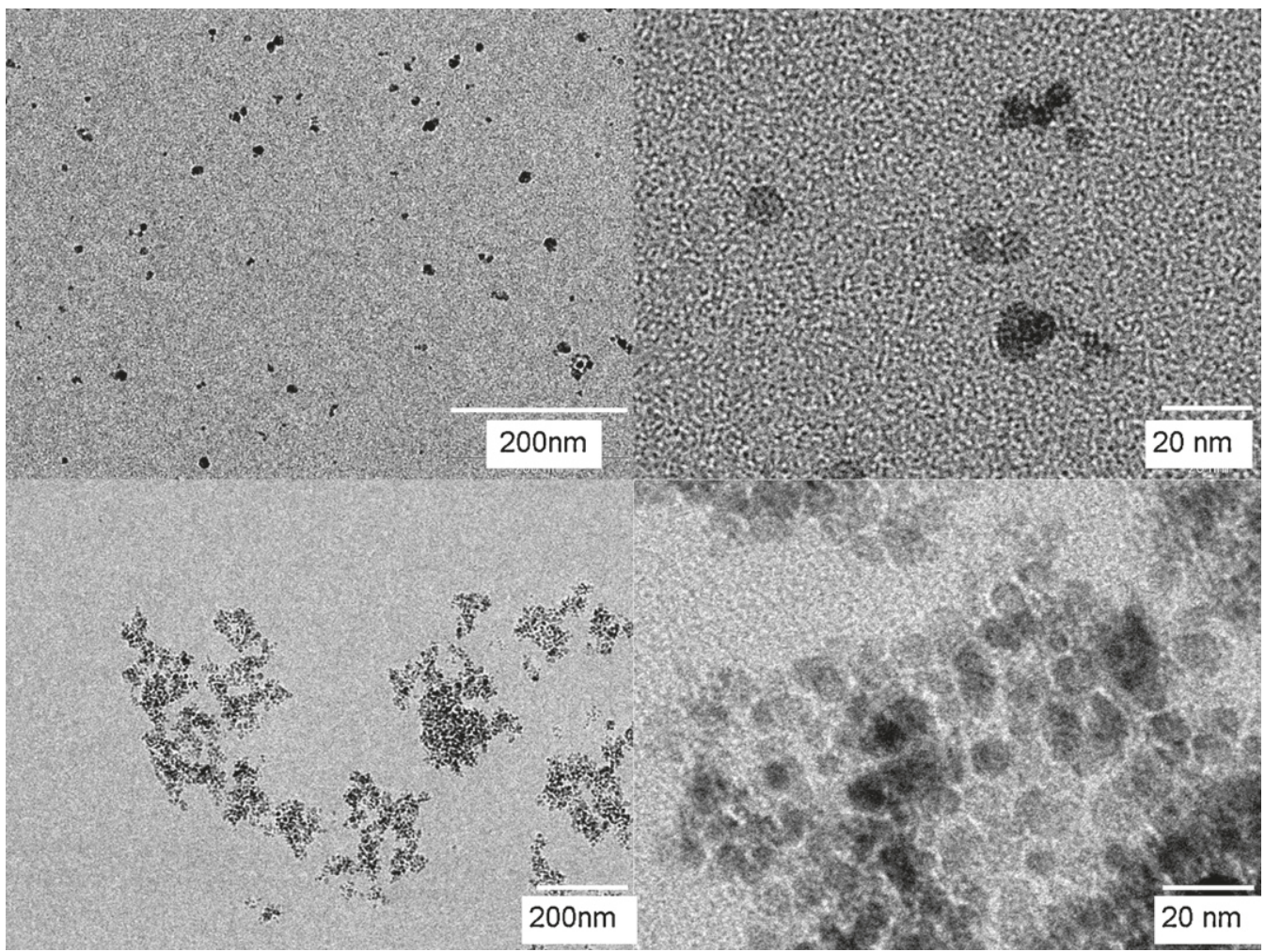

Fig. 6. TEM images of nanocomposites. Upper: $1 \%$ magnetite loading; bottom $2.5 \%$ magnetite loading.

Table 1

Hysteresis loops limits and blocking temperature for nanocomposites.

\begin{tabular}{|c|c|c|c|c|c|c|c|}
\hline \multirow[t]{2}{*}{$\% \mathrm{NP}, \mathrm{w}^{\mathrm{w}} \mathrm{w}^{-1}$} & \multicolumn{2}{|c|}{$M_{\mathrm{s}}\left(\mathrm{emu} \mathrm{g}^{-1}\right)$} & \multicolumn{2}{|c|}{$M_{\mathrm{r}}\left(\mathrm{emug}^{-1}\right)$} & \multicolumn{2}{|c|}{$H_{\mathrm{c}}(\mathrm{Oe})$} & \multirow[t]{2}{*}{$T_{\mathrm{B}}(\mathrm{K})$} \\
\hline & $300 \mathrm{~K}$ & $2 \mathrm{~K}$ & $300 \mathrm{~K}$ & $2 \mathrm{~K}$ & $300 \mathrm{~K}$ & $2 \mathrm{~K}$ & \\
\hline 0.25 & 0.044 & 0.081 & 0 & 0.047 & 0 & 285 & 63 \\
\hline 0.50 & 0.211 & 0.256 & 0 & 0.077 & 0 & 285 & 71 \\
\hline 1.00 & 0.360 & 0.496 & 0 & 0.189 & 0 & 285 & 84 \\
\hline 2.50 & 1.076 & 1.313 & 0 & 0.450 & 0 & 285 & 103 \\
\hline 5.00 & 2.233 & 2.626 & 0 & 0.900 & 0 & 285 & 105 \\
\hline 100.0 & 56.32 & 84.90 & 0 & 29.34 & 0 & 318 & 110 \\
\hline
\end{tabular}

nanocomposites with higher amount of magnetite. Nevertheless, superparamagnetic behavior with zero coercitivity and remanence at $300 \mathrm{~K}$ was observed at all the compositions, indicating that the particles on the agglomerates remain independent possibly due to the oleic acid layer which surrounds them. The limits of hysteresis loops are summarized at Table 1. The saturation magnetization is proportional to the amount of particles in the nanocomposite and, as expected, was higher at $2 \mathrm{~K}$ mainly due to spin disorder effect caused by thermal energy. The remanence at $2 \mathrm{~K}$ was found to be proportional to the amount of nanoparticles dispersed in the polymer matrix, although the coercitivity was constant for all samples suggesting a reduced influence of the polymer matrix on the magnetic properties of nanocomposite. The dependence of magnetization with temperature was measured in the same way as for the neat nanoparticles. All nanocomposites showed a maximum in the ZFC curve; the maximum shifts towards higher temperatures as the proportion of nanoparticles increases due to increased spin interactions originated by the decrease in the average distance between particles (Fig. 7). The blocking temperature in nanocomposites with high proportion of nanoparticles was close to the observed for the oleic acid modified nanoparticles. This effect could be related with

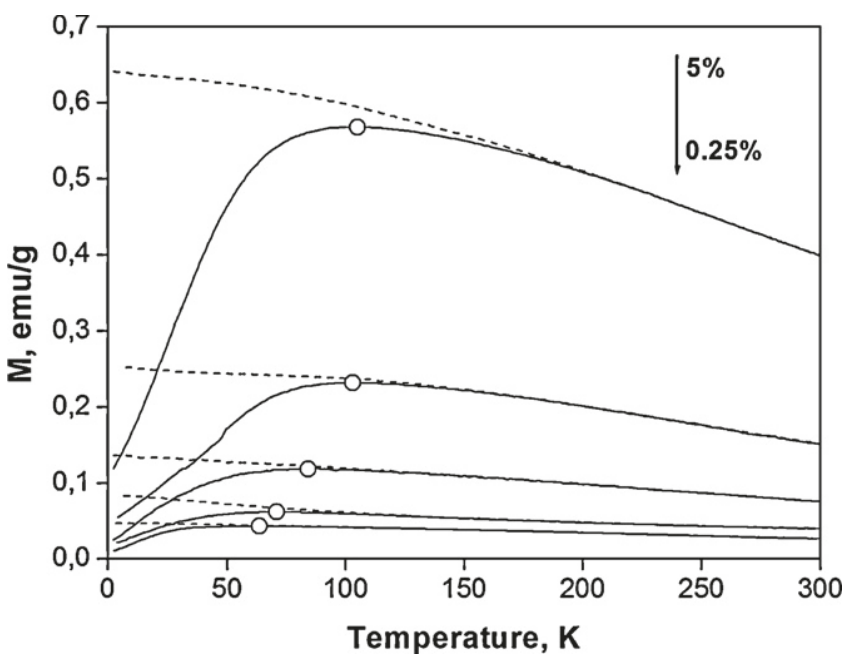

Fig. 7. ZFC (solid line) and FC (dashed line) curves for nanocomposites. 
Table 2

DSC and DMTA results for nanocomposites.

\begin{tabular}{lllll}
\hline$\% \mathrm{NP}$ & $T_{\mathrm{g}}\left({ }^{\circ} \mathrm{C}\right) \mathrm{DSC}$ & $T_{\mathrm{g}}\left({ }^{\circ} \mathrm{C}\right) \mathrm{DMTA}$ & $E_{\text {glass }}^{\prime}(\mathrm{MPa})$ & $E_{\text {rubber }}^{\prime}(\mathrm{MPa})$ \\
\hline 0 & 77.6 & 91 & 2600 & 12.0 \\
0.25 & 64.8 & 81 & 2641 & 10.2 \\
0.50 & 67.7 & 82 & 2829 & 11.7 \\
1.00 & 76.4 & 88 & 2553 & 11.8 \\
2.50 & 61.6 & 78 & 2842 & 10.5 \\
5.00 & 59.6 & 75 & 2829 & 10.4 \\
\hline
\end{tabular}

the formation of agglomerates in those systems with higher proportion of nanoparticles. In these cases the interactions between magnetic spins of the nanoparticles give rise to a similar behavior observed for bulk nanoparticles. Nevertheless all the samples showed a blockage temperature lower than the observed for magnetite nanoparticles.

Glass transitions temperature of all the composites was measured by Differential Scanning Calorimetry (DSC) and Dynamic Mechanical Thermal Analysis (DMTA) (Table 2). Since the principle of measurement of these two techniques is not the same, the $T_{\mathrm{g}}$ values obtained by DSC and DMTA are commonly different. In any case, the general behavior observed for the HDGEBA/magnetite nanocomposites is the same. Both techniques showed a single glass transition temperature which depends on the amount of nanoparticles. $T_{\mathrm{g}}$ values obtained by both techniques versus the nanoparticle content are shown in Fig. 8. Two tendencies can be clearly observed: $T_{\mathrm{g}}$ increases with nanoparticles content up to $1 \%$, and at higher magnetite contents (2.5\% and $5 \%$ ) it decreases. This behavior can be related with the dispersion degree of the nanoparticles in the epoxy matrix, which is high up to $1 \%$ while agglomerates are observed at higher loading. The $T_{\mathrm{g}}$ values obtained for the nanocomposites are lower than the measured for the neat epoxy $\left(T_{\mathrm{g}}=91^{\circ} \mathrm{C}\right.$ by DMTA). It is worthy to note that in this kind of nanocomposites there are two factors affecting $T_{\mathrm{g}}$ : the dispersion degree of the nanoparticles in the matrix and the presence of oleic acid chains which can act as plastifier of the cured network. This flexibilization effect of oleic acid has been previously reported for other oleic acid modified nanoparticles [54].

DMTA measurements did not show appreciable changes in $\tan \delta$ height or width of the transition measured by DMTA when incorporating nanoparticles to the epoxy matrix, indicating that there are no big changes in the homogeneity of the network. The storage modulus in the rubbery state remains constant independently of the amount of nanoparticles in the system, indicating that nanoparticles do not change crosslinking density. On the other

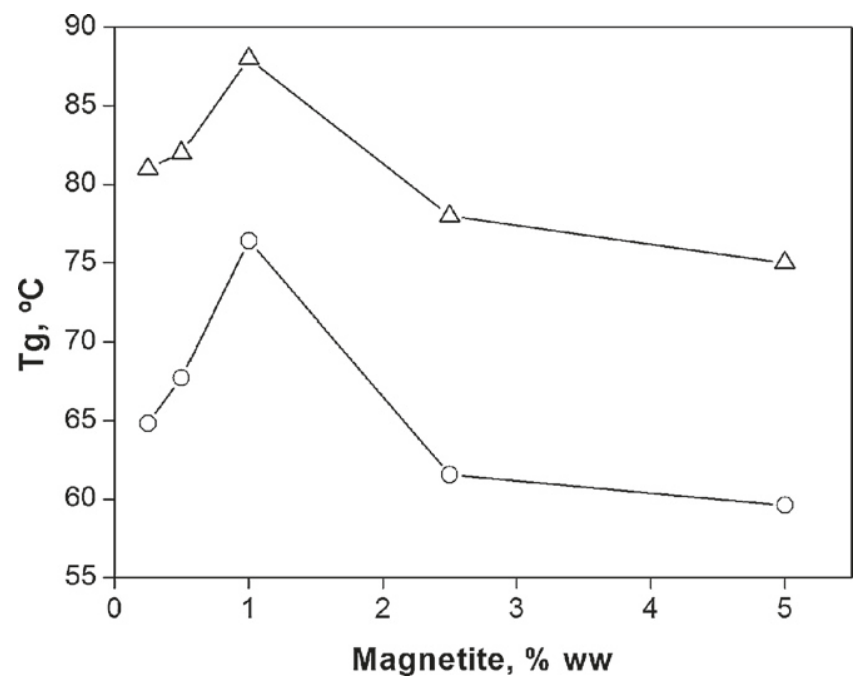

Fig. 8. $T_{\mathrm{g}}$ as a function of nanoparticles composition by DSC (- $\left.-^{-}\right)$and DMTA (- $\left.\Delta^{-}\right)$. hand, the glassy modulus slightly increases (10\%) with magnetite content. This behavior seems to indicate some reinforcement effect of magnetite particles on the epoxy matrix [55].

\section{Conclusions}

Magnetite nanoparticles modified with oleic acid were synthesized by co-precipitation of ferric and ferrous salts in ammonium solutions. The size of the nanoparticles was studied by DLS, TEM, XRD and magnetic measurements yielding values of: 16, 9.4, 9.4 and $9.3 \mathrm{~nm}$, respectively. The larger size obtained by DLS in stable dispersions in THF is due to the magnetite core plus the oleic layer and some solvent layers around it. The magnetic size is slightly lower than TEM size, due to the magnetic spin distortions originated in nanoparticles surface, and very close to the crystal size obtained by $\mathrm{XRD}$, indicating that the particle is fully crystalline. An oleic surface modification of $18.6 \%$ with only a $4.5 \%$ of physisorbed oleic acid was obtained by TGA, supported by FTIR measurements. These amounts are related with a surface monolayer of oleic on the nanoparticles. The diffraction signals obtained by XRD are characteristic of the magnetite spinel-like structure and no other phases (such as hematite) are observed. Field-cooling (FC) and zero-field-cooling (ZFC) at low magnetic field of 100 Oe shows a superparamagnetic behavior with a blocking temperature, $T_{\mathrm{B}}$, of $110 \mathrm{~K}$ and a splitting of curves at $150 \mathrm{~K}$. Superparamagnetic behavior was confirmed by hysteresis loops at 2 and $300 \mathrm{~K}$. Under $T_{\mathrm{B}}$, hysteresis losses appear in samples with a remanence and coercitive field of $30 \mathrm{emu} \mathrm{g}^{-1}$ and 293 Oe at $2 \mathrm{~K}$, while no hysteresis losses were observed with zero remanence and coercivity at $300 \mathrm{~K}$.

Thermosetting nanocomposites based in Magnetite:HDGEBA: m-xylilenediamine were prepared. Amounts of nanoparticles of $0.25,0.5,1,2.5$ and $5 \% \mathrm{ww}^{-1}$ were introduced via cosolvent magnetite:HDGEBA mixtures using THF. Good dispersion of nanoparticles was observed from TEM images with loadings up to $1 \%$ of magnetite. For higher contents agglomerates were observed although superparamagnetic behavior remained probably due to oleic surface modification. Remanence at $2 \mathrm{~K}$ increased with loading of nanoparticles, but the coercitivity was constant for all samples. This suggests a reduced influence of the polymer matrix on the magnetic nanocomposite properties. The blocking temperature obtained by ZFC curves increased with nanoparticle ratio mainly due to an increase of spin interactions induced by a decrease in the distances between particles. At high loadings blocking temperatures were close to bulk nanoparticles covered by oleic acid, probably due to the formation of agglomerates. No strong influence of the nanoparticles in thermal degradation behavior of the composite was observed, while thermal transitions showed two tendencies: for compositions below $1 \%$ of nanoparticles the glass transition temperature increased with composition; for higher compositions $T_{\mathrm{g}}$ decreased and this was attributed to the presence of agglomerates as observed by TEM.

\section{Acknowledgment}

This work was supported by grants Dinater (MAT2007-63722), Nacopan (MAT2007-31173-E) and Nanomod (MAT2010-17091) from the Spanish Ministerio de Ciencia e Innovación.

\section{References}

[1] F. Carruso, Adv. Mater. 13 (2001) 11-22.

[2] F. Hussain, M. Hojjati, M. Okamoto, R.E. Gorga, J. Compos. Mater. 40 (2006) 1511-1575.

[3] J. Jordan, K.I. Jaco, R. Tannenbaum, M.A. Sharaf, I. Jasiuk, Mater. Sci. Eng. A 393 (2005) $1-11$

[4] W. Huang, C.D. Han, Macromolecules 39 (2006) 257-267.[5]

V. Yong, H.T. Hahn, Nanotechnology 15 (2004) 1338-1343. 
[6] J.J. Mack, L.M. Viculis, A. Ali, R. Luoh, G. Yang, H.T. Hahn, F.K. Ko, R.B. Kaner, Adv. Mater. 17 (2005) 77-80.

[7] G. Sandi, H. Joachin, R. Kizilel, S. Seifert, K.A. Carrado, Chem. Mater. 15 (2003) 838-843.

[8] D. Makovec, A. Ko`sak, M. Drofenik, Nanotechnology 15 (2004) 160-166.

[9] V. Blaskov, V. Petkov, V. Rusanov, L.M. Martinez, B. Martinez, J.S. Mu noz, M. Mikhov, J. Magn. Magn. Mater. 162 (1996) 331-337.

[10] W. Ross, J. Am. Ceram. Soc. 63 (1980) 601-603.

[11] A. Wooding, M. Kilner, D.B. Lambrick, J. Colloid Interface Sci. 144 (1991) 236-242.

[12] D. Makovec, A. Ko`sak, M. Drofenik, Nanotechnology 15 (2004) S160-S166.

[13] M. Rozman, M. Drofenik, J. Am. Ceram. Soc. 78 (1995) 2449-2455.

[14] C.S. Kim, Y.S. Yi, K.T. Park, H. Namgung, J.G. Lee, J. Appl. Phys. 85 (1999) 5223-5225.

[15] S. Prasad, N.S. Gajbhiye, J. Alloys Compd. 265 (1998) 87-92.

[16] Y. Shi, J. Ding, X. Liu, J. Wang, J. Magn. Magn. Mater. 205 (1999) 249-254.

[17] T.YKaldés-Solís, P. Tartaj, G. Marbán, A.B. Fuertes, Nanotechnology 18 (2007)

[18] U. Häfeli, W. Schütt, J. Teller, M. Zborowski, Scientific and Clinical Applications of Magnetic Carriers, Plenum Press, New York, 1997, pp. 501-516.

[19] Q.A. Pankhurst, J. Connolly, S.K. Jones, J. Dobson, J. Phys. D: Appl. Phys. 36 (2003) R167-R181.

[20] A.K. Gupta, M. Gupta, Biomaterials 26 (2004) 3995-4021.

[21] L.P. Ramirez, K. Landfester, Macromol. Chem. Phys. 204 (2003) 22-31.

[22] R.A. Wassel, B. Grady, R.D. Kopke, K.J. Dormer, Colloids Surf. A: Physicochem. Eng. Aspects 292 (2007) 125-130.

[23] C.H. Peng, C.C. Hwang, J. Wan, J.S. Tsai, S.Y. Chen, Mater. Sci. Eng. B: Solid 117 (2005) 27-36

[24] X. Battle, A. Labara, J. Phys. D: Appl. Phys. 35 (2002) R15-R42.

[25] D.D.L. Chung, Carbon 39 (2001) 279-285.

[26] D.C. Culita, G. Marinescu, L. Patron, O. Carp, C.B. Cizmas, L. Diamandescu, Mater. Chem. Phys. 111 (2008) 381-385.

[27] C. Kittel, Phys. Rev. 70 (1946) 965-971.

[28] S. Kolev, A. Yanev, I. Nedkov, Phys. Stat. Sol. 3 (2006) 1308-1315.

[29] S. Gyergyek, M. Huskíc, D. Makovec, M. Drofenik, Colloids Surf. A: Physicochem. Eng. Aspects 317 (2008) 49-55.

[30] J. Zhang, D. Yu, W. Chen, Y. Xie, W. Wan, H. Liang, C. Min, J. Magn. Magn. Mater. 321 (2009) 572-577.

31] L. Jiang, W. Sun, J. Kim, Mater. Chem. Phys. 101 (2007) 291-296.

[32] P. Dallas, V. Georgakilas, D. Niarchos, P. Komninou, T. Kehagias, D. Petridis, Nanotechnology 17 (2006) 2046-2053.
[33] T. Banert, U.A. Peuker, J. Mater. Sci. 41 (2006) 3051-3056.

[34] I.G. Yá nez-Flores, R. Betancourt-Galindo, J.A. Matutes-Aquino, O. RodríguezFernández, J. Non-Cryst. Solids 353 (2007) 799-801.

[35] I. Kong, S.H. Ahmad, M.H. Abdullah, A.N. Yusoff, Proceedings of the nanoscience and nanotechnology, in: M. Rusop, T. Soga (Eds.), International Conference on Nanoscience and Nanotechnology (NANO-Sci-Tech 2008), AIP, 2009.

[36] S. Yang, H. Liu, Z. Zhang, J. Polym. Sci. Part A: Polym. Chem. 46 (2008) 3900-3910.

[37] M. Kobayashi, R. Matsuno, H. Otsuka, A. Takahara, Sci. Technol. Adv. Mater. 7 (2006) 617-628

[38] D. Ciprari, K. Jacob, R. Tannenbaum, Macromolecules 39 (2006) 6565-6573.[39] E.

Marutani, S. Yamamoto, T. Ninjbadgar, Y. Tsujii, T. Fukuda, M. Takano, Polymer 45 (2004) 2231-2235.

[40] X. Yu, S. Zhou, X. Zheng, T. Guo, Y. Xiao, B. Song, Nanotechnology 20 (2009) 235702.

[41] K.K. Yang, X.L. Wang, Y.Z. Wang, J. Ind. Eng. Chem. 13 (2007) 485-500.

[42] J.P. Lellouche, G. Senthil, A. Joseph, L. Buzhansky, I. Bruce, E.R. Bauminger, J. Schlesinger, J. Am. Chem. Soc. 127 (2005) 11998-12006.

[43] L.A. Ramajo, A.A. Cristóbal, P.M. Botta, J.M. Porto-López, M.M. Reboredo, M.S. Castro, Composites Part A 40 (2009) 388-393.

[44] A.N. Thorpe, F.E. Senftle, M. Holt, J. Grant, W. Lowe, H. Anderson, E. William, C. Monkres, A. Barkatt, J. Mater. Res. 15 (2000) 2488-2493.

[45] B. Hallouet, B. Wetzel, R. Pelster, J. Nanomater. (2007) 34527

[46] G. Kortaberria, P. Arruti, A.Jimeno, I. Mondragon, M. Sangermano, J. Appl. Polym. Sci. 109 (2008) 3224-3229.

[47] C.A. May, Epoxy Resins, Chemistry and Technology, Marcel Dekker, New York, 1988.

[48] LI. Klaassens, J. De Jong, M. Gillard, H. Van der Poel, European Patent EP 1359197A1 (2003).

[49] LI. Klaassens, J. De Jong, H. Van der Poel, European Patent EP 1359198A1 (2003).

[50] NR. Mowrer, RE. Foscante, L. Rojas, United States Patent US 5618860 (1997).[51]

NR. Mowrer, RE. Foscante, L. Rojas, United States Patent US 5804616 (1997)

[52] R.W. Chantrell, J. Popplewell, S.W. Charles, IEEE Trans. Magn. 14 (1978) 975-977.

[53] C. Caizer, J. Phys.: Condens. Matter 15 (2003) 765-776.

[54] M. Sangermano, I. Roppolo, G. Shan, M.P. Andrews, Prog. Org. Coat. 65 (2009) 431-434.

[55] Y.T. Sung, C.K. Kum, H.S. Lee, N.S. Byon, H.G. Yoon, W.N. Kim, Polymer 46 (2005) 5656-5661. 